\title{
EMS Systems in Lower-Middle Income Countries: A Literature
}

\section{Review}

Short running title: EMS Systems in Lower-Middle Income Countries

\section{Suryanto, M.Nurs; ${ }^{1,2}$ Virginia Plummer, RN, PhD; ; ${ }^{1,3}$ Malcolm Boyle,} MClinEpi, $\mathbf{P h D}^{4}$

1. School of Nursing and Midwifery, Monash University, Victoria, Australia

2. School of Nursing, Brawijaya University, Malang, Indonesia

3. Peninsula Health, Victoria, Australia

4. Department of Community Emergency Health and Paramedic Practice, Monash University, Victoria, Australia

\section{Correspondence:}

Suryanto, M.Nurs

[u1] $\mathrm{PhD}$ Candidate

School of Nursing and Midwifery

Monash University, Australia

Lecturer

School of Nursing

Brawijaya University, Indonesia 
E-mail: suryanto.s@monash.edu; suryanto.s@ub.ac.id

Conflicts of interest: none

Keywords: ambulance; EMS systems; lower-middle income countries; out-of-hospital;

prehospital

\section{Abbreviations:}

CPR: cardiopulmonary resuscitation

ECG: electrocardiogram

ED: emergency department

EMS: Emergency Medical Services

EMT: emergency medical technician

LMICs: lower-middle income countries

NAS: National Ambulance Services

UN: United Nations

VHWs: Village Health Workers

WB: World Bank

WHO: World Health Organization

Received: November 15, 2015

Revised: March 24, 2016

Accepted: May 29, 2016

\section{Abstract}


Introduction: Prehospital care is one of the many issues that require addressing by lowermiddle income countries (LMICs) where approximately 90\% of global injuries occur. This may arise from more traffic in LMICs, poor road conditions, lack of public awareness of the importance of road safety, and the lack of ability to provide first aid for the victims. However, prehospital care in LMICs remains underdeveloped.

Problem: There is insufficient evidence regarding the development of prehospital care among LMICs. Thus, the objective of this study was to investigate the status of Emergency Medical Services (EMS) systems in these countries.

Methods: A review of medical-related electronic databases was designed to identify the development of EMS systems in LMICs. A search of the literature was undertaken using three electronic databases, CINAHL, Ovid Medline, and EMBASE via Ovid, from their commencement date until the end of July2015. The grey literature was searched using Google Scholar. Articles were included if they reported on the establishment and current status of an EMS system and were excluded if they were letters to the editor, articles focusing on disaster management, a combination of more than one country if the other country was not LMIC, written in a language other than English or Bahasa Indonesia, and/or focusing only on inhospital care.

Results: There were 337 articles identified in CINAHL, 731 in Ovid Medline, 891 in Embase via Ovid, and 41 in Google Scholar. Based on the title and abstract, 31 articles from CINAHL, 40 from Ovid Medline, 43 from EMBASE, and 11 from Google Scholar were retrieved for further review. There were 92 articles that met the inclusion criteria with 35 articles removed, as they were duplicated, leaving 57 articles to be reviewed. From those 48 countries categorized as LMICs, there were 16 (33.3\%) countries that had information about an EMS system, including injury types, patient demographic, prehospital transport, and the obstacles in implementing the prehospital care system. 
Conclusion: The implementation and development of an EMS system is varied among LMICs. Many LMICs lack an organized EMS system with most ambulances used purely for transport and not as an emergency care vehicle. Financial issues are the most common problems faced by LMICs with support from developed countries a necessity.

\section{Suryanto, Plummer V, Boyle M. EMS systems in lower-middle income countries: a literature review. Prehosp Disaster Med. 20XX;YY(Z):mm-nn.}

\section{Introduction}

Prehospital care is one of the many issues that require addressing by lower-middle income countries (LMICs). Even though communicable diseases remain the focus of the health sector in many countries, trauma and accidents have been increasing in these countries. ${ }^{1,2}$ This may arise from greater use of vehicles in LMICs, poor road conditions, and also a lack of public awareness of the importance of road safety and the inability to provide first aid for the victims. ${ }^{3}$ Based on World Health Organization (WHO; Geneva, Switzerland) data, 90\% of global injuries occur in LMICs and are the cause of approximately 5.8 million deaths annually. ${ }^{4}$ Poor prehospital care might be one of the causes of this high number of deaths; this is because the morbidity and mortality of road traffic accidents can be reduced by establishing well-organized prehospital care/Emergency Medical Services (EMS) $)^{5-7}$ and trauma care facilities. ${ }^{8,9}$ Furthermore, the prehospital emergency care is not always needed by traumatic and obstetric patients, ${ }^{4}$ but non-traumatic emergency patients, such as communicable and non-communicable disease patients, also require prehospital care, including transport to hospital. ${ }^{10}$ Based on the importance of prehospital care, it is necessary to establish an organized EMS system; however, prehospital care in LMICs has been, and still is, underdeveloped. ${ }^{11}$ 
There are several methods for categorizing countries based on their development. The United Nations (UN; New York, New York USA), World Bank (WB; Washington, DC USA), and WHO have different categorization systems. The UN categorizes countries based on Gross National Income, Human Assets Index, and the Economic Vulnerability Index. ${ }^{12}$ Based on the UN criteria, there are three categories: developed economies, economies in transition, and developing economies. The WB categorizes countries based on region and income. For the income criteria, there are low-income countries, lower-middle income countries, upper-middle income countries, and high-income countries. ${ }^{13}$ The WHO report describes the countries based on nine indicators of global health, including countries listed alphabetically by regions and income groups with additional information. ${ }^{14}$ Similar to the WHO report, this report will use the categorization from the WB. Examples of low-income countries include Afghanistan and Cambodia, where lower-middle income countries include Indonesia and India, upper-middle income countries include Mexico and Malaysia, and highincome countries include Australia, United Kingdom, and the United States. ${ }^{13}$

In this report, issues related to the status of an EMS system in LMICs will be described. The countries included as LMICs were based on the WB categorization process and included 48 countries (Table 1). The objective of this study was to investigate the development and status of EMS systems in LMICs.

\section{Report}

Study Design

This was a review of medical-related electronic databases to identify EMS systems in LMICs. 
A search was undertaken using the electronic medical databases CINAHL Plus (EBSCO Information Services; Ipswich, Massachusetts USA), EMBASE (Elsevier; Amsterdam, Netherlands), and Ovid Medline (Ovid Technologies; New York, New York USA) from their commencement date until the end of July 2015. In order to add to the credibility and validity of the review, the search also included grey literature using Google Scholar (Google Inc.; Mountain View, California USA). The search strategy used the following keywords: “prehospital”, “pre hospital”, “pre-hospital”, “out-of-hospital”, “out of hospital”, “pre hospital care”, “EMS”, “EMT”, “ambulance”, “ambulance service”, “EMT”, “paramedic”, "paramedics", and the 48 countries categorized as LMICs. The search processing used the keywords individually and in combination.

Articles were included if they reported on the establishment of an EMS system, including the funding of the system, the human resources for the EMS system, the prehospital patient demographics, the case load and incident type, prehospital transport mode (type of vehicle and staff configuration), availability of the ambulance, and the obstacles of implementing the EMS system. Articles were excluded if they were not in English or Bahasa Indonesia, letters to editors, focusing on disaster management, a combination of more than one country where at least one country was not LMIC, and focusing only on in-hospital care or emergency department (ED) care.

\section{Results}

Based on the 13 keywords used for the search, there were 31,110 articles identified in CINAHL, 48,748 in Ovid Medline, 60,789 in EMBASE via Ovid, and 1,108 articles in Google Scholar. Those results were then combined with 48 countries and generated 337 articles in CINAHL, 731 articles in Ovid Medline, 891 articles in EMBASE via Ovid, and 41 articles from Google Scholar. Based on the title and abstract, there were 31 articles from 
CINAHL, 40 from Ovid Medline, 43 from EMBASE via Ovid, and 11 articles from Google Scholar retrieved for further review. After matching based on the inclusion and exclusion criteria, there were 92 articles that met the criteria with 35 duplicated articles removed; these left 57 articles to be reviewed. There were no further articles found from the review of the reference list of the retrieved articles. From those 48 countries categorized as LMICs, there were 16 countries (33.3\%) that had information about an EMS system. The process of identifying and retrieving the articles is demonstrated in Figure 1 and the summary of the findings can be found in Table 2 .

\section{Discussion}

This is the first time a literature review has been undertaken to identify the establishment and management of an EMS system in LMICs. Overall, there is a lack of current information about EMS systems in LMICs, and that the information available identified a lack of efficient EMS systems in LMICs.

\section{Availability of the EMS System}

The availability of an EMS system among LMICs is varied. Most LMICs lack an organized EMS system, for example, Indonesia, India, Morocco, Vietnam, Armenia, Nigeria, Ghana, and Sri Lanka. ${ }^{5,15-22}$ Indonesia implemented an EMS system during the establishment of the Safe Community Program in 2000. The program was implemented in order to guarantee that the citizens are safe and healthy by establishing 118 ambulance services in 18 cities in Indonesia. ${ }^{16}$ The progress of EMS development in Indonesia seems slow-moving due to financial issues. ${ }^{16}$

The 115 emergency call center was launched in 2007 in Morocco, but it was not protected by any legislation and there was no evaluation of the effectiveness of the system. ${ }^{17}$ 
The cost of calling the emergency call center is US\$0.20 with the call received by an intensive care/anesthetics intern station in a hospital ED. The system in Sri Lanka was piloted in 2009 in the Anuradhapura, north central region of Sri Lanka, ${ }^{23}$ but there is no published article describing the current development of the system in Sri Lanka.

In 1997, Vietnam had no organized EMS system and an ambulance was used for “transport” only. ${ }^{18}$ The scoop-and-run approach was used in order to transport the injured patient to hospital using any available vehicle. In 2004, the situation remained the same; an ambulance was rarely used for transporting patients but was used for transporting honored guests. ${ }^{24}$ However, there was a positive change seen in 2010 when there were Village Health Workers (VHWs) and health volunteers ${ }^{25}$ used in an EMS role. Nguyen el at ${ }^{25}$ interviewed 3,000 of the VHWs to assess their capacity. Approximately 31\% of the VHWs and volunteers were trained by local hospitals to manage injuries with $16 \%$ of them equipped with essential emergency equipment.

India does not have a country-wide organized EMS system. In 2002, there was a project initiated to establish an EMS system in the country. ${ }^{26}$ However, there were several challenges in establishing an EMS system in India, such as a lack of public awareness and financial issues. ${ }^{26}$ In 2008, Kumar et $\mathrm{al}^{27}$ investigated the knowledge, attitude, and practice in EMS among resident doctors, consultants, and private practitioners. The study showed that participants had less than adequate knowledge and practice in EMS. In 2010, the EMS system was still unorganized with $43.5 \%$ of injured patients being helped by a passer-by and no one waiting for an ambulance. ${ }^{15}$ Another similar study also showed that $66.1 \%$ patients were transported by private car and not by ambulance. ${ }^{28}$ The initial 108 Emergency Service in India was established in Andhra Pradesh and managed by GVK Emergency Management and Research Institute (GVK EMRI; Andhra Pradesh, India). ${ }^{29}$ Then, the project was supported by local government, and in 2011, the other nine states adopted the same model and operated 
around 2,300 ambulances in those ten states. ${ }^{29}$ However, prehospital care in India remains steady where prehospital care coordination only occurs in metropolitan areas and is nonexistent in urban and semi-rural areas with no national guidelines or protocol covering prehospital care. ${ }^{9}$ The establishment of the private ambulance system has become one of EMS system development in India. The Ziqitza Healthcare Limited (Mumbai, India), established in 2005, is one of the private EMS systems in the country. ${ }^{30}$ It had a call center called 1298 in Mumbai and started the system with 10 ambulances; then in 2009, the system was expanded to the other two states, Alleppey and Kottayam, with the addition of five Advanced Life Support (ALS) and 10 Basic Life Support (BLS) ambulances. ${ }^{30}$

According to Ahidjo et al, Nigeria also lacks an organized prehospital care system and has obsolete trauma centers and less-qualified trauma teams. ${ }^{5}$ In 2003, the nearest ambulance service in some parts of Ghana was over 100 miles away and there was no dispatch center for coordination and dispatching of an ambulance. ${ }^{31}$ Then in 2004, the EMS system in Ghana, called the National Ambulance Service (NAS), was established by providing seven pilot ambulance stations in collaboration with the Fire Service and other hospital-based ambulances; by 2014, NAS was available in every district of the country. ${ }^{32}$ In Yerevan, Armenia, the city had the 103 emergency call center with one dispatch center and eight subcenters in $2013 .{ }^{33}$ The EMS system in the city involved 650 staff members and 200 physicians with 35 ambulances; 10 of the ambulances were resuscitation ambulances. ${ }^{33}$ There was a similar situation in Khartoum, Sudan. The state had one central, 999 call center, and involved 37 ambulances and 29 hospitals. ${ }^{34}$

The Ukraine was part of the Soviet Union, and the EMS systems appears to be well organized as a result. ${ }^{35}$ The Ukraine gained independence from the Soviet Union in 1991, with the Ukraine taking over the EMS system, which was founded in $1902 .{ }^{35}$ The EMS system in the Ukraine was one of the first systems in the world to perform home 
electrocardiograms (ECGs), prehospital thrombolysis, and prehospital defibrillation. Definitive care is often conducted in the field and it is not expected that the patient will be transported to hospital routinely. The research was undertaken in three main Ukrainian cities, Kiev, Vinnitsya, and Gnivan. Most of the EMS systems are computerized with nurses as the dispatchers.

Pakistan established an EMS system in the mid-2000s. Rescue 1122 was launched in 2004 and was centered in the Punjab state. ${ }^{11}$ The system has now been implemented in other provinces in Pakistan. Karachi, the largest city in Pakistan, has decentralized ambulance services which were operated by non-profit organizations or were hospital-based. ${ }^{36}$ There were two ambulance services in Karachi, the Edhi Foundation and Aman Foundation ambulance services. ${ }^{36}$ The Edhi Foundation, established in 1955, provides ambulances with no trained staff nor emergency equipment inside the ambulance; while the Aman Foundation, established in 2009, provides ambulance services with medical equipment and trained medical technicians, drivers, and doctor upon request. ${ }^{36}$ Nevertheless, there are seven keys of a successful EMS system in Pakistan: (1) providing a legislative framework; (2) human resources management; (3) establishing an organizational structure; (4) strategic planning of ambulance station locations; (5) organizing prehospital care; (6) expanding the scope of service; and (7) quality monitoring. ${ }^{11}$ By applying these methods, the EMS can maintain an average seven minute response time in all 35 districts within the Punjab state. ${ }^{11}$ The success of Pakistan in establishing an EMS system is due to several reasons: (1) using local manufacturing products and equipment, especially for the vehicles; (2) strategic planning for ambulance station locations, which is based on response time rather than target population; (3) assistance from developed countries; (4) adopting the teaching and training to meet local needs; and (5) using a single command structure for all first responders, ambulance, rescue, and fire services. ${ }^{11}$ 


\section{Funding}

Funding is one of the main obstacles in establishing a well-organized EMS system. Since most LMICs do not have an organized EMS system, most of the prehospital care cost is a citizen's responsibility. ${ }^{15}$ Due to the high cost of using prehospital care services, people in Ghana seek other forms of transport and do not seek help from medical institutions but seek to use traditional healing methods. ${ }^{31}$ Even in the Ukraine, with its comparatively wellorganized EMS system compared to other LMICs, after the breakup of the Soviet Union, the Ukraine still has a low budget for maintaining the EMS system, approximately US\$4 million annually. ${ }^{35}$ The government in Sri Lanka is not the only source of funds for the developing EMS system. The EMS system in Sri Lanka was developed by support from other sources such as Sarvodaya (a community-based organization; Moratuwa, Sri Lanka), Red Cross (Colombo, Sri Lanka), private hospitals, private ambulance service, and foreign government aid. $^{22}$

Assistance from developed countries is necessary to enhance the quality of prehospital care in LMICs. There was support for the Punjab state in Pakistan from the United States and United Kingdom in establishing their EMS system. To support the staffing of the EMS system in the Punjab, the United States provided four months of training for more than 6,000 rescuers costing almost US $\$ 1.5$ million. ${ }^{11}$ Pakistan also expanded the scope of service into the fire services with assistance from the United Kingdom, which provided modern fire appliances and formal training for firemen. ${ }^{11}$ Another advantage gained from international support is the Sri Lankan first responders/paramedics can take the Australasian Registry of Emergency Medical Technicians (EMTs) regional exam because the EMT training is provided by the US. ${ }^{22}$ Similarly, India had support from the George Washington University (Washington, DC USA) in the US, which assisted in launching the first EMS 
system in 2002. This support consisted of EMT class teaching, designing and procuring ambulances, equipment for the health clinic, and a field delivery management system. ${ }^{26}$

\section{Human Resources: Availability}

Human resources are varied among LMICs, and some employ physicians, nurses, or paramedics; however, there is only a driver in the ambulance in certain countries. Most LMICs lack the appropriate human resources for efficiently organized prehospital care and systems. Even though some countries already have training, most LMIC prehospital care providers are unable to provide it. ${ }^{15}$ In Morocco, even though an ambulance is staffed by a driver and two paramedics, only one out of three is trained in prehospitalcare. ${ }^{17}$ Similar to Morocco, EMS staff in Nigeria also are inexperienced and overworked, ${ }^{5}$ and emergency assistants employed in the ambulances in Khartoum, Sudan had limited medical training. ${ }^{34}$ In Pakistan, each ambulance includes a driver and two EMTs. ${ }^{11}$ In 1997, an ambulance in Vietnam included a driver who had little or no ability to manage patients in the prehospital setting, ${ }^{18}$ while in 2010, the availability of VHWs and health volunteers had improved the prehospital care available. Ten percent of VHWs had experience in providing care to road traffic injured patients and $36.7 \%$ had experience in medical emergency care. ${ }^{25}$ Similar to Vietnam, ambulance staffing in Nicaragua consists of a driver with no training, who was paid less than US\$100 per month for three to four 24-hour shifts a week, ${ }^{21}$ with no further information regarding other staff for ambulance services.

In contrast, the Ukraine ambulance staff includes specially trained physicians and nurses. ${ }^{35}$ The EMS dispatchers are nurses and a physician who is always present in the dispatch center. Depending on the area, there are 26 to 145 prehospital teams consisting of

physician-nurse teams or nurse-nurse teams, which are available 24 hours a day. ${ }^{35}$ There are a large number of employees paid a low salary in the Ukraine. 
What can be learned from other countries is the empowering of the community. The VHWs and health volunteers in Vietnam can enhance the quality of prehospital care. It is not only laypersons that can be empowered in the community; military medics also can be empowered. In the Sudan, 40 hours of training was undertaken to prepare the military medics as prehospital care providers. ${ }^{37}$ The training had a positive influence in improving participants' knowledge and skills, especially in wound care, caring for the patient with a hemorrhage, and managing the patient with head trauma.

\section{Education for EMS Personnel}

The education system for prehospital care providers is varied among the different countries. In Morocco, there is a paramedic school consisting of two years basic training and one year on-the-job training with $50 \%$ of the paramedics having resuscitation skill training. ${ }^{17}$ In Armenia, there was no formal training in emergency medicine or prehospital care for EMS staff. ${ }^{19}$ Most of the EMS providers in Armenia did on-the-job training and developed their own style and approach of learning; thus, their expertise is by experience. ${ }^{19}$ In Ghana, EMS personnel were trained in cardiopulmonary resuscitation (CPR), oxygen therapy, bandaging, and splinting, but without any formal certification. ${ }^{31}$ Even though there are no data regarding prehospital education in Sri Lanka, the knowledge of EMTs in the country was good. ${ }^{38}$

As mentioned previously, support from developed countries is essential in improving the knowledge and skills of EMS personnel by providing training in prehospital care. Based on Pakistan’s experience, support from developed countries can include training in prehospital care, emergency dispatch, documentation, plus water and fire rescue. ${ }^{11}$ Similarly, support from the George Washington University in the US allowed India to implement EMT education as an initial step to establishing the EMS system in 2002. The initial focus was on 
basic CPR, patient assessment, lifting and moving, anatomy and physiology, safety, and cardiology. ${ }^{26}$

\section{The Case and Type of Transportation}

Trauma cases are the major incidents in LMICs; the majority of prehospital trauma cases were related to traffic incidents..$^{3,20,24,28,39}$ In Armenia, 38\% of cases were hip fracture, 31\% knife wounds, and $29.5 \%$ neuroses and psychosis. ${ }^{19}$ Fifty percent of trauma cases were traffic-related injury in Ghana and falls were the second highest of the prehospital cases. ${ }^{20}$ This was similar in India ${ }^{28}$ where $73 \%$ of accidents in India were motorbikers and $14 \%$ of them were influenced by alcohol. ${ }^{28}$ Burn injuries also accounted for high incidents among LMICs. $^{20,28}$ In the majority of trauma incidents, between $60 \%$ and $90 \%$ of patients were males $^{7,15,40-45}$ in their 30s. ${ }^{28,42,43}$ In Ghana, most patients were transported by taxi from the scene to the hospital, ${ }^{20,31}$ while in India and Nigeria, most patients were transported by private car. ${ }^{5,28}$ In Pakistan, which has a more developed prehospital care system, the ambulance was the most used prehospital transportation in the country. ${ }^{39}$ Most patients were transported within an hour of the incident to the nearest ED. ${ }^{20,43}$

\section{Characteristic of the Ambulances}

The characteristics and effectiveness of the ambulances varied among LMICs. In 2000, La Trinidad in Nicaragua had two ambulances and one untrained driver. ${ }^{21}$ One ambulance was an old white pick-up truck which was used primarily for transport, and another ambulance was donated by UNICEF (New York, New York USA), which was more suitable but still without oxygen or suction equipment. In Morocco, the ambulance is used for transporting severe trauma patients with three out of 15 ambulances equipped with resuscitation equipment and one ambulance well-equipped for use during major social/sporting events. ${ }^{17}$ 
The other 12 ambulances were ordinary ambulances which were used for transport and equipped with first-aid equipment only. In the Ukraine, most ambulances were equipped with a cardiac monitor/defibrillator with some ambulances using an ECG machine which could transmit the ECG to the hospital from the scene. ${ }^{35}$ However, in Gnivan, another part of the Ukraine, there was only one older ambulance which had no cardiac monitor/defibrillator, and other equipment, medication, and trauma and obstetric boxes were stored at the base station and would be taken to the ambulance as needed. ${ }^{35}$

\section{Obstacles}

The establishment of a well-organized EMS system in LMICs had many obstacles. Financial resources and a poverty level of income are the two biggest problems. ${ }^{16,23,26,31,46}$ Lack of funding may lead to insufficient medication, supplies, and equipment for the services and a large out-of-pocket payment by community. ${ }^{46}$ Thirty four percent of people involved in a study of the services in India mentioned that they would not call an ambulance because it cost too much. ${ }^{30}$ The lack of public awareness for the need for an EMS system also influences the implementation of an EMS system. ${ }^{26,47}$ A study in Ghana found that only 22 (4.5\%) people involved in the study had ever called an ambulance for emergency cases because $77.4 \%$ of them believed that a taxi was faster than an ambulance. ${ }^{48}$ Lack of general emergency knowledge among the society, including performing CPR, is one of the issues faced by Armenia. ${ }^{46}$ Cultural issues where people believe that any accident or diseases is the "will of God” must be understood and accepted, ${ }^{16}$ along with the people’s belief and use of more traditional healing methods compared to modern health care. ${ }^{31}$ In Ghana, people (23\% of respondents in the study) believed that ambulances were used to transport corpses rather than emergency patients. ${ }^{49}$ This misperception may have led to low ambulance utilization in Ghana. ${ }^{49}$ The condition of the roads, the traffic volume, and lack of road infrastructure are 
also obstacles to prehospital care in LMICs. ${ }^{31,46,47}$ The summary of the obstacles faced by LMICs in implementing EMS system can be seen in Table 3.

\section{Limitations}

This study is potentially limited by several articles found being more than 10 years old. It is possible that the situations have changed in these countries; therefore, the current situation in most LMICs remains unknown. The study also is potentially limited by the exclusion of nonEnglish articles, as some theoretically relevant articles may have been missed.

\section{Conclusion}

Little is known regarding EMS systems of LMICs, but for those with published data, the review conclusions can be made. The implementation of an EMS system is varied among LMICs and dependent on the characteristic of each country. In general, based on information published in the databases reviewed, most LMICs lack an organized EMS system with most ambulances used only for transport and not as an emergency care vehicle. The most advanced EMS system was implemented by the Ukraine, which resulted following the break-up of the Soviet Union. The least advanced countries have no EMS system and rely on an unorganized system of private cars or other transport to take patients to hospital. Financial issues and lack of training of staff are the most common problems faced by LMICs, with support from developed countries a necessity for the sustainability of the system.

\section{References}

1. Tiska MA, Adu-Ampofo M, Boakye G, Tuuli L, Mock CN. A model of prehospital trauma training for lay persons devised in Africa. Emerg Med J. 2004;21(2):237-239. 
2. Mock CN, Adzotor KE, Conklin E, Denno DM, Jurkovich GJ. Trauma outcomes in the rural developing world: comparison with an urban level I trauma center. $J$ Trauma. 1993;35(4):518-523.

3. Jena BN, Dubey A, Dhal M. Challenges in handling medical emergencies by ambulance drivers: a comparative study in two metropolitan cities in India. J Emerg Manag. 2010;8(1):9.

4. Nielsen K, Mock C, Joshipura M, Rubiano AM, Zakariah A, Rivara F. Assessment of the status of prehospital care in 13 low- and middle-income countries. Prehosp Emerg Care. 2012;16(3):381-389.

5. Ahidjo KA, Olayinka SA, Ayokunle O, Mustapha AF, Sulaiman GAA, Golahan AT. Prehospital transport of patients with spinal cord injury in Nigeria. J Spinal Cord Med. 2011;34(3):308-311.

6. Joshipura MK. Trauma care in India: current scenario. World J Surg. 2008;32(8):16131617.

7. Mould-Millman CN, Rominski S, Oteng R. Ambulance or taxi? High acuity prehospital transports in the Ashanti region of Ghana. African J Emerg Med. 2014.

8. Tahir N, Naseer R, Khan SM, Macassa G, Hashmi W, Durrani M. Road traffic crashes managed by Rescue 1122 in Lahore, Pakistan. Int J Inj Contr Saf Promot. 2012;19(4):347350.

9. Oestern HJ, Garg B, Kotwal P. Trauma care in India and Germany. Clin Orthop Relat Res. 2013;471(9):2869-2877.

10. Sasson C, Keirns CC, Smith D, et al. Small area variations in out-of-hospital cardiac arrest: does the neighborhood matter? Ann Intern Med. 2010;153(1):19-22. 
11. Waseem H, Naseer R, Razzak JA. Establishing a successful prehospital emergency service in a developing country: experience from Rescue 1122 service in Pakistan. Emerg Med J. 2011;28(6):513-515.

12. United Nations. World Economic Situations and Prospects 2012. http://www.un.org/en/development/desa/policy/wesp/wesp_archive/2012wesp.pdf. Accessed September 12, 2013.

13. World Bank. World Bank List of Economies (July 2013). http://siteresources.worldbank.org/DATASTATISTICS/Resources/CLASS.XLS. Accessed October 7, 2013.

14. World Health Organization. World Health Statistics 2013. http://www.who.int/ gho/publications/world_health_statistics/2013/en/. Accessed July 30, 2013.

15. Roy N, Murlidhar V, Chowdhury R, et al. Where there are no Emergency Medical Services - prehospital care for the injured in Mumbai, India. Prehosp Disaster Med. 2010;25(2):145-151.

16. Pusponegoro AD. Terrorism in Indonesia. Prehosp Disaster Med. 2003;18(2):100-105.

17. Tachfouti N, Bhatti JA, Nejjari C, Kanjaa N, Salmi LR. Emergency trauma care for severe injuries in a Moroccan region: conformance to French and World Health Organization standards. J Healthc Qual. 2011;33(1):30-38.

18. Richards JR. Emergency medicine in Vietnam. Ann Emerg Med. 1997;29(4):543-545.

19. Becker BM, Handrigan MT, Jagminas L, Becker TJ. Emergency Medical Services in the reconstruction phase following a major earthquake: a case study of the 1988 Armenia earthquake. PrehospDisaster Med. 1998;13(1):28-33.

20. Forjuoh SN, Mock CM, Friedman DI, Quansah R. Transport of the injured to hospitals in Ghana: the need to strengthen the practice of trauma care. Prehosp Immed Care. 1999;3:66-70. 
21. Berman J. International EMS. Third world EMS: US Peace Corp volunteer experiences EMS - Nicaraguan style. Emerg Med Serv. 2000;29(4):76-78.

22. Hesser F. From the ground up: a young American helps Sri Lanka construct a prehospital care system. EMS Mag. 2008;37(8):121.

23. Dassanayake L. Challenges faced in establishing the emergency prehospital ambulance service in north central Sri Lanka: developing something from nothing. PrehospDisaster Med. 2011;26(Supplement 1):s94-s95.

24. Bailey K, Ansell T. Nursing together: Australian and Vietnam: trauma nursing in a developing country. AustralEmerg Nurs J. 2004;7(1):23-27.

25. Nguyen TH, Ngoc LTT, Luong MA. Assessing injury emergency response of village health workers and volunteers in five provinces in Vietnam. Inj Prev. 2010;16:A76.

26. Patrick RW. International EMS. An EMS system for India. Emerg Med Serv. 2002;31(6):106-110.

27. Kumar S, Agarwal AK, Kumar A, Agrawal GG, Chaudhary S, Dwivedi V. A study of knowledge, attitude, and practice of hospital consultants, resident doctors, and private practitioners with regard to prehospital and emergency care in Lucknow. Indian J Surg. 2008;70(1):14-18.

28. Uthkarsh PS. Study of gaps between precepts and practices in prevention of injuries and prehospital care among injury cases admitted to MSRMC Bangalore, Karnataka, India. Inj Prev. 2010;16:A195.

29. Jena BN, Umar NS. Emergency Medical Services (EMS) In India - effectiveness inmanaging morbidities resulting from accidents, injuries, poisoning, fracture,and burns. Indian Emerg J. 2011;6(1):31-38. 
30. Gupta R, Basu S. Ziqitza healthcare limited: challenge of scaling up Emergency Medical Services (EMS) in India using public-private partnership (PPP) mode. Asian J Manag Cas. 2014;11(1):5-21.

31. Smith CR. International EMS. EMS in Ghana. Emerg Med Serv. 2003;32(1):87-88.

32. Frimpong J, Dinye RD. Ambulance emergency services and healthcare provision in Ghana: adistrict level performance appraisal. Int JHealth Med Info. 2014;3(1):13-23.

33. Baghdassarian A. Knowledge and Attitudes Assessment of Out-of-Hospital Emergency Physicians in Yerevan, Armenia. http://jdc.jefferson.edu/mphcapstone_presentation/89/. Accessed March 10, 2016.

34. Elbashir K, Gore R, Bloem C, et al. Prehospital emergency care in Sudan - current practices in disaster management. Prehosp Disaster Med. 2011;26(Suppl 1):s89.

35. Wright SW, Stack LB, McMurray BR, Bolyukh S. Emergency medicine in Ukraine: challenges in the post-Soviet era. Am J Emerg Med. 2000;18(7):828-832.

36. Chandran A, Ejaz K, Karani R, Baqir M, Razzak J, Hyder AA. Insights on the effects of patient perceptions and awareness on ambulance usage in Karachi, Pakistan. Emerg Med J. 2014;31:990-993.

37. VanRooyen MJ, Erickson TB, Cruz C, Levy P, Isaacs JK. Training military medics as civilian prehospital care providers in southern Sudan. Prehosp Emerg Care. 2000;4(1):6569.

38. Nandasena LGS, Abeysena C. Quality of ambulance care available for transfer of emergency patients to the National Hospital of Sri Lanka. J College Comm Phys Sri Lanka. 2009;14(Sup 1):21.

39. Waseem H, Carenzo L, Razzak J, Naseer R. Epidemiology of major incidents: an EMS study from Pakistan. Int J Emerg Med. 2011;4:48. 
40. Jain A, Menezes RG, Kanchan T, Gagan S, Jain R. Two wheeler accidents on Indian roads--a study from Mangalore, India. J Forensic Leg Med. 2009;16(3):130-133.

41. Espitia-Hardeman V, Rocha J, Clavel-Arcas C, Dahlberg L, Mercy JA, Concha-Eastman A. Characteristics of non-fatal injuries in Leon, Nicaragua - 2004. Int JInj Contr Saf Promot. 2007;14(2):69-75.

42. Oluwadiya KS, Olakulehin AO, Olatoke SA, et al. Pre-hospital care of the injured in South Western Nigeria: a hospital based study of four tertiary level hospitals in three states. Annu ProcAssoc Adv Automot Med. 2005;49:93-100.

43. Solagberu BA, Ofoegbu CK, Abdur-Rahman LO, Adekanye AO, Udoffa US, Taiwo J. Pre-hospital care in Nigeria: a country without Emergency Medical Services. Nigerian J Clin Pract. 2009;12(1):29-33.

44. Farooqui JM, Chavan KD, Bangal RS, et al. Pattern of injury in fatal road traffic accidents in a rural area of western Maharashtra, India. Aust Med J. 2013;6(9):476-482.

45. Razzak JA, Laflamme L. Limitations of secondary data sets for road traffic injury epidemiology: a study from Karachi, Pakistan. PrehospEmerg Care. 2005;9(3):355-360.

46. Truzyan N. Barriers for better Emergency Medical Services in Armenia trigger distrust of the general population towards services. http://eurpub.oxfordjournals.org/ content/eurpub/24/suppl_2/cku161.022.full.pdf. Accessed March 10, 2016.

47. Pitt E, Pusponegoro A. Prehospital care in Indonesia. Emerg Med J. 2005;22(2):144-147.

48. Mould-Millman NK, Rominski SD, Zakariah AN, Akoriyea SK, Bogus J, Boatemaah CA. Barriers to accessing Emergency Medical Services in Ghana. Ann Emerg Med. 2014;1:S71-S72.

49. Mould-Millman NK, Rominski S, Bogus J, et al. Barriers to accessing Emergency Medical Services in Accra, Ghana: development of a survey instrument and initial application in Ghana. Glob Health Sci Pract. 2015;3(4):557-590. 


\begin{tabular}{|l|l|l|l|}
\hline Armenia & Guatemala & Mongolia & Sri Lanka \\
\hline Bhutan & Guyana & Morocco & Sudan \\
\hline Bolivia & Honduras & Nicaragua & Swaziland \\
\hline Cameroon & India & Nigeria & Syrian Arab Republic \\
\hline Cape Verde & Indonesia & Pakistan & Timor-Leste \\
\hline Congo, Rep. & Kiribati & Papua New Guinea & Ukraine \\
\hline Cote d'Ivoire & Kosovo & Paraguay & Uzbekistan \\
\hline Djibouti & Lao PDR & Philippines & Vanuatu \\
\hline Egypt, Arab Rep. & Lesotho & Samoa & Vietnam \\
\hline El Salvador & Mauritania & Sao Tome and Principe & West Bank and Gaza \\
\hline Georgia & Micronesia, Fed. Sts. & Senegal & Yemen, Rep. \\
\hline Ghana & Moldova & Solomon Islands & Zambia \\
\hline
\end{tabular}

Table 1. Low-Middle Income Countries

\begin{tabular}{|l|c|c|c|c|l|}
\hline \multicolumn{1}{|c|}{$\begin{array}{c}\text { Information } \\
\text { Available }\end{array}$} & \multicolumn{2}{c|}{ No. of Articles } & \multicolumn{2}{c|}{ No. of Countries } & \multicolumn{1}{c|}{ Countries } \\
\cline { 2 - 5 } EMS System & $\mathbf{n}$ & $\mathbf{\%}$ & $\mathbf{n}$ & $\mathbf{\%}$ & \\
\hline Human Resources & 19 & 33.3 & 12 & 25 & $\begin{array}{l}\text { Armenia, Ghana, Guatemala, India, } \\
\text { Indonesia, Kosovo, Morocco, Pakistan, } \\
\text { Philippines, Sudan, Ukraine, Vietnam. }\end{array}$ \\
\hline Patient Demographic & 20 & 35.1 & 6 & 12.5 & $\begin{array}{l}\text { Ghana, India, Indonesia, Nicaragua, Nigeria, } \\
\text { Pakistan. }\end{array}$ \\
\hline Injury Type/Case & 23 & 40.4 & 7 & 14.6 & $\begin{array}{l}\text { Armenia, Ghana, India, Indonesia, } \\
\text { Nicaragua, Nigeria, Pakistan. }\end{array}$ \\
\hline Prehospital Transport & 11 & 19.3 & 4 & 8.3 & Ghana, India, Nigeria, Pakistan. \\
\hline $\begin{array}{l}\text { Prehospital } \\
\text { Education/Training }\end{array}$ & 3 & 5.3 & 2 & 4.2 & Armenia, India. \\
\hline Transfer/Referral & 4 & 7.1 & 3 & 6.2 & Ghana, India, Nigeria. \\
\hline Mortality & 9 & 15.8 & 4 & 8.3 & Ghana, India, Nigeria, Vietnam. \\
\hline Funding & 4 & 7.1 & 3 & 6.2 & India, Pakistan, Sri Lanka. \\
\hline Obstacles/Challenges & 13 & 22.8 & 5 & 10.4 & Armenia, Ghana, India, Indonesia, Sri Lanka. \\
\hline
\end{tabular}

Table2. Summary of Information Regarding Prehospital Care among LMICs with Published Data

Abbreviations: EMS, Emergency Medical Services; LMICs, low-middle income countries.

\begin{tabular}{|l|c|c|c|c|l|}
\hline \multirow{2}{*}{ Information Available } & \multicolumn{2}{|c|}{ No. of Countries } & \multicolumn{2}{|c|}{ No. of Articles } & \multicolumn{1}{c|}{ Countries } \\
\cline { 2 - 5 } Financial Problem & $\mathbf{n}$ & $\mathbf{\%}$ & $\mathbf{n}$ & $\mathbf{\%}$ & \\
\hline Lack of Public Awareness & 4 & 8.3 & 6 & 8.8 & $\begin{array}{l}\text { Armenia, Ghana, India, Indonesia, } \\
\text { Sri Lanka. }\end{array}$ \\
\hline Cultural Issues & 4 & 8.3 & 4 & 7.1 & Armenia, Ghana, India, Indonesia. \\
\hline Infrastructure & 2 & 4.2 & 3 & 5.3 & Ghana, Indonesia. \\
\hline
\end{tabular}

Table 3. Obstacles in Implementing EMS system in LMICs

Abbreviations: EMS, Emergency Medical Services; LMICs, low-middle income countries.

Figure 1. Articles Retrieved from Three Databases. 